\title{
Dynamic Analysis of Stochastic Lotka-Volterra Predator-Prey Model with Discrete Delays and Feedback Control
}

\author{
Jinlei Liu (iD ${ }^{1}$ and Wencai Zhao (D) $^{1,2}$ \\ ${ }^{1}$ College of Mathematics and Systems Science, Shandong University of Science and Technology, Qingdao 266590, China \\ ${ }^{2}$ State Key Laboratory of Mining Disaster Prevention and Control Co-founded by Shandong Province and the Ministry of Science \\ and Technology, Shandong University of Science and Technology, Qingdao 266590, China
}

Correspondence should be addressed to Wencai Zhao; zhaowencai@sdust.edu.cn

Received 2 June 2019; Revised 26 September 2019; Accepted 24 October 2019; Published 16 November 2019

Academic Editor: Mahdi Jalili

Copyright ( 2019 Jinlei Liu and Wencai Zhao. This is an open access article distributed under the Creative Commons Attribution License, which permits unrestricted use, distribution, and reproduction in any medium, provided the original work is properly cited.

\begin{abstract}
In this paper, a stochastic Lotka-Volterra predator-prey model with discrete delays and feedback control is studied. Firstly, the existence and uniqueness of global positive solution are proved. Further, we investigate the asymptotic property of stochastic system at the positive equilibrium point of the corresponding deterministic model and establish sufficient conditions for the persistence and extinction of the model. Finally, the correctness of the theoretical derivation is verified by numerical simulations.
\end{abstract}

\section{Introduction}

In nature, time delays exist in many ecosystems [1-5]. For example, maturity stage is a common phenomenon in biological population, and many diseases have a long incubation period. The mathematical model describing this phenomenon with time delay is called the delay differential equation. In 1999, Saito et al. [6] studied a Lotka-Volterra predator-prey model with discrete delays, which can be defined as follows:

$$
\left\{\begin{array}{l}
\frac{\mathrm{d} x(t)}{\mathrm{d} t}=x(t)\left[r_{1}+a x(t)+\alpha x\left(t-\tau_{1}\right)-\beta y\left(t-\tau_{2}\right)\right], \\
\frac{\mathrm{d} y(t)}{\mathrm{d} t}=y(t)\left[r_{2}+a y(t)+\beta x\left(t-\tau_{1}\right)+\alpha y\left(t-\tau_{2}\right)\right],
\end{array}\right.
$$

where $x(t)$ and $y(t)$ stand for the population density of prey and predator at time $t$, respectively. $r_{i}(i=1,2)$ represent the intrinsic growth rate of corresponding population. $\tau_{1}$ and $\tau_{2}$ are discrete time delays. $a(a<0), \alpha$ and $\beta$ are constants.

Due to the environmental changes and increased human activities, many rare species are at risk of extinction. How to protect endangered species of floras and faunas and maintain the diversity of ecosystems is an important issue that needs to be solved urgently. In the process of marine fishery production, overfishing often results in the exhaustion of fishery resources. It is rewarding for humans to develop and utilize the ecological system of the population rationally, which also contributes to the sustainability of the system [7-14]. In 2003, Gopalsamy and Weng [15] studied the following population competition model with feedback control: 


$$
\left\{\begin{array}{l}
\frac{\mathrm{d} x_{1}(t)}{\mathrm{d} t}=x_{1}(t)\left[b_{1}-a_{11} x_{1}(t)-a_{12} x_{2}(t)-\alpha_{1} u_{1}(t-\tau)\right] \\
\frac{\mathrm{d} x_{2}(t)}{\mathrm{d} t}=x_{2}(t)\left[b_{2}-a_{21} x_{1}(t)-a_{22} x_{2}(t)-\alpha_{2} u_{2}(t-\tau)\right], \\
\frac{\mathrm{d} u_{1}(t)}{\mathrm{d} t}=-\eta_{1} u_{1}(t)+a_{1} x_{1}(t-\tau), \\
\frac{\mathrm{d} u_{2}(t)}{\mathrm{d} t}=-\eta_{2} u_{2}(t)+a_{2} x_{2}(t-\tau),
\end{array}\right.
$$

where $u_{1}(t)$ and $u_{2}(t)$ are the feedback control variables, $b_{i}>0, a_{i j}>0, \alpha_{i}>0, \eta_{i}>0$, and $a_{i}>0(i, j=1,2)$. They also discussed the existence of positive equilibrium point and global attraction of the model. In 2013, Li et al. [16] introduced feedback control variables into the twospecies competition system and discussed the extinction and global attraction of equilibrium points. They found that if the two-species competition model is globally stable, the system retains the stable property after adding feedback controls and the position of equilibrium point is changed. If the two-species competition model is extinct, by choosing the suitable values of feedback control variables, they can make extinct species become globally stable, or still keep the property of extinction. In 2017, Shi et al. [17] discussed a LotkaVolterra predator-prey model with discrete delays and feedback control as follows:

$$
\left\{\begin{array}{l}
\frac{\mathrm{d} x_{1}(t)}{\mathrm{d} t}=x_{1}(t)\left[r_{1}-a_{11} x_{1}(t)-a_{12} x_{2}\left(t-\tau_{1}\right)\right] \\
\frac{\mathrm{d} x_{2}(t)}{\mathrm{d} t}=x_{2}(t)\left[r_{2}+a_{21} x_{1}\left(t-\tau_{2}\right)-a_{22} x_{2}(t)-c u(t)\right] \\
\frac{\mathrm{d} u(t)}{\mathrm{d} t}=-e u(t)+f x_{2}(t)
\end{array}\right.
$$

where $u(t)$ is the feedback control variable, $e$ and $f$ denote the feedback control coefficients, $a_{i i}(i=1,2)$ denote the intraspecific competition rates, $a_{i j}(i \neq j, i, j=1,2)$ stand for the capturing rates of the prey and predator populations, $\tau_{1}$ is the time of catching prey, and $\tau_{2}$ is maturation delay of predator. Shi et al. [17] show that

(i) The solution $\left(x_{1}(t), x_{2}(t), u(t)\right)$ of system (3) is ultimately bounded (ii) When the conditions $\left(r_{1} / r_{2}\right)>\left(a_{12} /\left(a_{22}+(c f / e)\right)\right)$, $\left(a_{11} / a_{21}\right)>\left(a_{12} / a_{22}\right)$ are established, system (3) has a unique globally asymptotically stable positive equilibrium point $\left(x_{1}^{*}, x_{2}^{*}, u^{*}\right)$, where $x_{1}^{*}=\left(e\left(r_{1} a_{22}\right.\right.$ $\left.\left.-r_{2} a_{12}\right)+r_{1} c f\right) /\left(e\left(a_{11} a_{22}+a_{12} a_{21}\right)+c f a_{11}\right), x_{2}^{*}=$ $e\left(r_{2} a_{11}+r_{1} a_{21}\right) /\left(e\left(a_{11} a_{22}+a_{12} a_{21}\right)+c f a_{11}\right), \quad$ and $u^{*}=(f / e) x_{2}^{*}$

In fact, in nature, ecosystems are inevitably affected by various environmental noises [18-28]. Mathematical models with environmental disturbances can usually be described by stochastic differential equations. Stochastic noise can generally be divided into two categories: one type is a small number of strong interference, usually called colored noise or electrical noise, which can be described by the Markov chain [29-31]; the other type is the sum of many small, independent random interference, called white noise, which is usually represented by Brownian motion [32-35]. Assume that the population's intrinsic growth rate $r_{i}$ is disturbed by white noise:

$$
r_{i} \longrightarrow r_{i}+\sigma_{i} \dot{B}_{i}(t), \quad i=1,2 .
$$

Then, model (3) is transformed into

$$
\left\{\begin{array}{l}
\mathrm{d} x_{1}(t)=x_{1}(t)\left[r_{1}-a_{11} x_{1}(t)-a_{12} x_{2}\left(t-\tau_{1}\right)\right] \mathrm{d} t \\
\quad+\sigma_{1} x_{1}(t) \mathrm{d} B_{1}(t) \\
\mathrm{d} x_{2}(t)=x_{2}(t)\left[r_{2}+a_{21} x_{1}\left(t-\tau_{2}\right)-a_{22} x_{2}(t)-c u(t)\right] \mathrm{d} t \\
\quad+\sigma_{2} x_{2}(t) \mathrm{d} B_{2}(t) \\
\mathrm{d} u(t)=\left[-e u(t)+f x_{2}(t)\right] \mathrm{d} t
\end{array}\right.
$$

and satisfies the initial conditions

$$
\begin{array}{ll}
x_{i}(\theta)=\phi_{i}(\theta), & \\
u(\theta)=\psi(\theta), & \\
& \theta \in[-\tau, 0], \\
& i=1,2,
\end{array}
$$

where $B_{i}(t)(i=1,2)$ denote the independent standard Brownian motion, $\sigma_{i}^{2}$ denote the intensity of white noise, $\tau=\max \left\{\tau_{1}, \tau_{2}\right\}, \phi_{i}(0)>0, \psi(0)>0$, and $\phi_{i}(\theta)$ and $\psi(\theta)$ are both nonnegative continuous functions on $[-\tau, 0]$.

Due to the interference of stochastic noise, system (5) does not possess an equilibrium point. An interesting question is: Does model (5) still have stability? What is the influence of white noise on system (5)? This paper mainly studies the dynamical properties of stochastic systems (5) also satisfying initial conditions (6). The second part proves the suitability of the system. The third part discusses the oscillation of the stochastic model near the positive equilibrium point $\left(x_{1}^{*}, x_{2}^{*}, u^{*}\right)$ of the corresponding 
deterministic model. The fourth and fifth parts, respectively, obtain the conditions for the persistence and extinction of the stochastic system. Finally, the correctness of the theoretical derivation is verified by numerical simulation.

\section{Existence and Uniqueness of Global Positive Solutions}

The stochastic differential equation is expressed as

$$
\mathrm{d} x(t)=f(x(t), t) \mathrm{d} t+g(x(t), t) \mathrm{d} B(t), x \in R^{n} .
$$

If the Lyapunov function $V(x, t) \in C^{2,1}\left[R^{n} \times R_{+}, R\right]$, the stochastic differential equation of $V(x, t)$ along system (7) is defined as [36]

$$
\begin{aligned}
\mathrm{d} V(x(t), t)= & {\left[V_{t}(x(t), t)+V_{x}(x(t), t) f(x(t), t)\right.} \\
& \left.+\frac{1}{2} \operatorname{tr}\left(g^{T}(x(t), t) V_{x x}(x(t), t) g(x(t), t)\right)\right] \mathrm{d} t \\
& +V_{x}(x(t), t) g(x(t), t) \mathrm{d} B(t) \text { a.s, }
\end{aligned}
$$

where $L V=V_{t}(x, t)+V_{x}(x, t) f(x, t)+(1 / 2) \operatorname{tr}\left(g^{T}(x, t)\right.$ $\left.V_{x x}(x, t) g(x, t)\right)$ represent diffusion operator.

Theorem 1. For any given initial condition (6), model (5) has a unique global positive solution $\left(x_{1}(t), x_{2}(t), u(t)\right)$, and the solution will remain in $\mathbb{R}_{+}^{3}$ with probability one.

Proof. Since the coefficients of system (5) satisfy the locally Lipschitz condition, for any given initial condition (6), model (5) has a unique local positive solution $\left(x_{1}(t), x_{2}(t)\right.$, $u(t))$ in interval $t \in\left[0, \tau_{e}\right)$, where $\tau_{e}$ is the explosion time.

To prove that this solution is global, we only need to prove $\tau_{e}=\infty$ a.s. Let $k_{0}>0$ be a sufficiently large constant for any initial value $x_{1}(0), x_{2}(0)$, and $u(0)$ lying within the internal $\left[\left(1 / k_{0}\right), k_{0}\right]$. For each integer $k \geq k_{0}$, define the stopping time

$$
\begin{aligned}
\tau_{k}= & \inf \left\{t \in\left[0, \tau_{e}\right): \min \left\{x_{1}(t), x_{2}(t), u(t)\right\}\right. \\
& \left.\leq \frac{1}{k} \text { or } \max \left\{x_{1}(t), x_{2}(t), u(t)\right\} \geq k\right\} .
\end{aligned}
$$

Obviously, $\tau_{k}$ is increasing as $k \longrightarrow \infty$. Let $\tau_{\infty}=$ $\lim _{k \longrightarrow \infty} \tau_{k}$; therefore, $\tau_{\infty} \leq \tau_{e}$ a.s. Now, we need to verify $\tau_{\infty}=\infty$ a.s. Otherwise, there are two constants $T>0$ and $\epsilon \in(0,1)$ such that $P\left\{\tau_{\infty} \leq T\right\}>\epsilon$. So, there is a positive integer $k_{1} \geq k_{0}$, such that

$$
P\left\{\tau_{k} \leq T\right\}>\epsilon, \quad k \geq k_{1} .
$$

Define a $C^{2}$ - function $V: \mathbb{R}_{+}^{3} \longrightarrow \mathbb{R}_{+}$by

$$
\begin{aligned}
V\left(x_{1}, x_{2}, u\right)= & x_{1}-1-\ln x_{1}+\frac{a_{11} a_{22}}{a_{21}^{2}}\left(x_{2}-1-\ln x_{2}\right) \\
& +\frac{1}{2} a_{11} \int_{t-\tau_{2}}^{t} x_{1}^{2}(s) \mathrm{d} s \\
& +a_{12} \int_{t-\tau_{1}}^{t} x_{2}(s) \mathrm{d} s+\frac{a_{11} a_{22} c}{2 f a_{21}^{2}} u^{2}+u-1-\ln u \\
= & V_{1}\left(x_{1}, x_{2}\right)+V_{2}(u),
\end{aligned}
$$

where

$$
\begin{aligned}
V_{1}\left(x_{1}, x_{2}\right)= & x_{1}-1-\ln x_{1}+\frac{a_{11} a_{22}}{a_{21}^{2}}\left(x_{2}-1-\ln x_{2}\right) \\
& +\frac{1}{2} a_{11} \int_{t-\tau_{2}}^{t} x_{1}^{2}(s) \mathrm{d} s+a_{12} \int_{t-\tau_{1}}^{t} x_{2}(s) \mathrm{d} s, \\
V_{2}(u)= & \frac{a_{11} a_{22} c}{2 f a_{21}^{2}} u^{2}+u-1-\ln u .
\end{aligned}
$$

The nonnegativity of this function can be obtained from

$$
x-1-\ln x \geq 0, \quad x>0 .
$$

Applying Itô's formula yields

$$
\begin{aligned}
\mathrm{d} V\left(x_{1}, x_{2}, u\right)= & L V\left(x_{1}, x_{2}, u\right) \mathrm{d} t+\sigma_{1}\left(x_{1}-1\right) \mathrm{d} B_{1}(t) \\
& +\frac{a_{11} a_{22}}{a_{21}^{2}} \sigma_{2}\left(x_{2}-1\right) \mathrm{d} B_{2}(t),
\end{aligned}
$$

where 


$$
\begin{aligned}
& L V=L V_{1}+L V_{2}, \\
& L V_{1}=\left(x_{1}(t)-1\right)\left[r_{1}-a_{11} x_{1}(t)-a_{12} x_{2}\left(t-\tau_{1}\right)\right]+\frac{1}{2} \sigma_{1}^{2}+\frac{a_{11} a_{22}}{a_{21}^{2}}\left(x_{2}(t)-1\right)\left[r_{2}+a_{21} x_{1}\left(t-\tau_{2}\right)-a_{22} x_{2}(t)-c u(t)\right] \\
& +\frac{a_{11} a_{22}}{2 a_{21}^{2}} \sigma_{2}^{2}+\frac{1}{2} a_{11} x_{1}^{2}(t)-\frac{1}{2} a_{11} x_{1}^{2}\left(t-\tau_{2}\right)+a_{12} x_{2}(t)-a_{12} x_{2}\left(t-\tau_{1}\right) \\
& =\left(r_{1}+a_{11}\right) x_{1}(t)-\frac{1}{2} a_{11} x_{1}^{2}(t)-a_{12} x_{1}(t) x_{2}\left(t-\tau_{1}\right)-r_{1}+\frac{1}{2} \sigma_{1}^{2}+\frac{a_{11} a_{22}}{a_{21}^{2}}\left[\left(r_{2}+a_{22}\right) x_{2}(t)-a_{22} x_{2}^{2}(t)-c x_{2}(t) u(t)+c u(t)\right] \\
& +\frac{a_{11} a_{22}}{a_{21}}\left(x_{2}(t)-1\right) x_{1}\left(t-\tau_{2}\right)+\frac{a_{11} a_{22}}{a_{21}^{2}}\left(-r_{2}+\frac{1}{2} \sigma_{2}^{2}\right)-\frac{1}{2} a_{11} x_{1}^{2}\left(t-\tau_{2}\right)+a_{12} x_{2}(t) \\
& \leq\left(r_{1}+a_{11}\right) x_{1}(t)-\frac{1}{2} a_{11} x_{1}^{2}(t)+\frac{a_{11} a_{22}}{a_{21}^{2}}\left[r_{2} x_{2}(t)-\frac{1}{2} a_{22} x_{2}^{2}(t)-c x_{2}(t) u(t)+c u(t)\right]+a_{12} x_{2}(t)-r_{1}+\frac{1}{2} \sigma_{1}^{2} \\
& +\frac{a_{11} a_{22}}{a_{21}^{2}}\left(-r_{2}+\frac{1}{2} \sigma_{2}^{2}+\frac{1}{2} a_{22}\right) \\
& L V_{2}=\frac{a_{11} a_{22} c}{f a_{21}^{2}} u(t)\left(-e u(t)+f x_{2}(t)\right)+\left(1-\frac{1}{u(t)}\right)\left(-e u(t)+f x_{2}(t)\right) \\
& \leq-\frac{a_{11} a_{22} c e}{f a_{21}^{2}} u^{2}(t)+\frac{a_{11} a_{22} c}{a_{21}^{2}} u(t) x_{2}(t)-e u(t)+f x_{2}(t)+e .
\end{aligned}
$$

Therefore,

$$
\begin{aligned}
L V= & L V_{1}+L V_{2} \\
\leq & \left(r_{1}+a_{11}\right) x_{1}(t)-\frac{1}{2} a_{11} x_{1}^{2}(t)+\frac{a_{11} a_{22}}{a_{21}^{2}}\left[r_{2} x_{2}(t)-\frac{1}{2} a_{22} x_{2}^{2}(t)-c x_{2}(t) u(t)+c u(t)\right]+a_{12} x_{2}(t)-r_{1} \\
& +\frac{1}{2} \sigma_{1}^{2}+\frac{a_{11} a_{22}}{a_{21}^{2}}\left(-r_{2}+\frac{1}{2} \sigma_{2}^{2}+\frac{1}{2} a_{22}\right)-\frac{a_{11} a_{22} c e}{f a_{21}^{2}} u^{2}(t)+\frac{a_{11} a_{22} c}{a_{21}^{2}} u(t) x_{2}(t)-e u(t)+f x_{2}(t)+e \\
\leq & \max \left\{-\frac{1}{2} a_{11} x_{1}^{2}(t)+\left(r_{1}+a_{11}\right) x_{1}(t)\right\}+\frac{a_{11} a_{22}}{a_{21}^{2}} \max \left\{-\frac{1}{2} a_{22} x_{2}^{2}(t)+\left(r_{2}+\frac{a_{21}^{2}\left(f+a_{12}\right)}{a_{11} a_{22}}\right) x_{2}(t)\right\} \\
& +\frac{a_{11} a_{22}}{a_{21}^{2}} \max \left\{-\frac{c e}{f} u^{2}(t)+\left(c-\frac{a_{21}^{2} e}{a_{11} a_{22}}\right) u(t)\right\}+\frac{a_{11} a_{22}}{a_{21}^{2}}\left(\frac{1}{2} a_{22}+\frac{1}{2} \sigma_{2}^{2}-r_{2}\right)+\frac{1}{2} \sigma_{1}^{2}+e-r_{1}
\end{aligned}
$$

$\leq K$,

where $K$ is a positive constant. So, we get

$$
\mathrm{d} V \leq K \mathrm{~d} t+\sigma_{1}\left(x_{1}-1\right) \mathrm{d} B_{1}(t)+\frac{a_{11} a_{22}}{a_{21}^{2}} \sigma_{2}\left(x_{2}-1\right) \mathrm{d} B_{2}(t)
$$

Integrating (17) from 0 to $\tau_{k} \wedge T$ and taking expectation on both sides, we have

$$
\begin{array}{r}
E V\left(x_{1}\left(\tau_{k} \wedge T\right), x_{2}\left(\tau_{k} \wedge T\right), u\left(\tau_{k} \wedge T\right)\right) \\
\leq E V\left(x_{1}(0), x_{2}(0), u(0)\right)+K T .
\end{array}
$$

Set $\Omega_{k}=\left\{\tau_{k} \leq T\right\}$, and from inequality (10), we have $P\left(\Omega_{k}\right) \geq \epsilon$. Note that, for every $\omega \in \Omega_{k}$, there is at least one of $x_{1}\left(\tau_{k}, \omega\right), x_{2}\left(\tau_{k}, \omega\right)$, or $u\left(\tau_{k}, \omega\right)$ equaling either $k$ or $(1 / k)$, and then, we have 


$$
\begin{gathered}
V\left(x_{1}\left(\tau_{k} \wedge T\right), x_{2}\left(\tau_{k} \wedge T\right), u\left(\tau_{k} \wedge T\right)\right) \\
\quad \geq(k-1-\ln k) \wedge\left(\frac{1}{k}-1-\ln \frac{1}{k}\right) .
\end{gathered}
$$

It can be obtained by (18)

$$
\begin{aligned}
& E V\left(x_{1}(0), x_{2}(0), u(0)\right)+K T \geq E\left[1_{\Omega_{k}(\omega)} V\left(x_{1}\left(\tau_{k}, \omega\right), x_{2}\left(\tau_{k}, \omega\right), u\left(\tau_{k}, \omega\right)\right)\right] \\
& \quad \geq \varepsilon(k-1-\ln k) \wedge\left(\frac{1}{k}-1+\ln k\right),
\end{aligned}
$$

where $1_{\Omega_{k}}$ is the indicator function of $\Omega_{k}$, and letting $k \longrightarrow \infty$ yields

$$
\infty>E V\left(x_{1}(0), x_{2}(0), u(0)\right)+K T=\infty .
$$

This is a contradiction; we must have $\tau_{\infty}=\infty$, and we have completed the proof.

$$
\left(A_{1}\right): \frac{r_{1}}{r_{2}}>\frac{a_{12}}{a_{22}+(c f / e)}, \frac{a_{11}}{a_{21}}>\frac{a_{12}}{a_{22}} .
$$

Theorem 2. For any given initial condition (6), if hypothesis $\left(A_{1}\right)$ is established, the solution $\left(x_{1}(t), x_{2}(t), u(t)\right)$ of system (5) has the property that

$$
\begin{aligned}
& \limsup _{t \rightarrow+\infty} \frac{1}{t} E \int_{0}^{t}\left[\left(x_{1}(\theta)-x_{1}^{*}\right)^{2}+\left(x_{2}(\theta)-x_{2}^{*}\right)^{2}\right. \\
& \left.\quad+\left(u(\theta)-u^{*}\right)^{2}\right] \mathrm{d} \theta \leq \frac{n}{m},
\end{aligned}
$$

(5) will have stochastic oscillation. Next, we discuss the asymptotic property of stochastic system at the positive equilibrium point of the corresponding deterministic model. In order to study the problem conveniently, the hypothesis is

where

$$
\begin{aligned}
& m=\min \left\{\frac{a_{11} f\left(a_{11} a_{22}-a_{12} a_{21}\right)}{a_{11} a_{22}+a_{12} a_{21}}, \frac{a_{12} f\left(a_{11} a_{22}-a_{12} a_{21}\right)}{2 a_{11} a_{21}}, \frac{a_{12} c e}{a_{21}}\right\}, \\
& n=\frac{f x_{1}^{*} \sigma_{1}^{2}}{2}+\frac{a_{12} f x_{2}^{*} \sigma_{2}^{2}}{2 a_{21}},
\end{aligned}
$$

where $\left(x_{1}^{*}, x_{2}^{*}, u^{*}\right)$ is the positive equilibrium point of the corresponding deterministic model (3).

Proof. Define the function

$$
\begin{aligned}
V\left(x_{1}, x_{2}, u\right)= & f\left(x_{1}-x_{1}^{*}-x_{1}^{*} \ln \frac{x_{1}}{x_{1}^{*}}\right)+\frac{a_{12} f}{a_{21}}\left(x_{2}-x_{2}^{*}-x_{2}^{*} \ln \frac{x_{2}}{x_{2}^{*}}\right)+\frac{a_{12} c}{2 a_{21}}\left(u-u^{*}\right)^{2} \\
& +\frac{a_{12} f}{2 \omega_{2}} \int_{t-\tau_{2}}^{t}\left(x_{1}(s)-x_{1}^{*}\right)^{2} \mathrm{~d} s+\frac{a_{12} f \omega_{1}}{2} \int_{t-\tau_{1}}^{t}\left(x_{2}(s)-x_{2}^{*}\right)^{2} \mathrm{~d} s,
\end{aligned}
$$

where $\omega_{1}$ and $\omega_{2}$ are positive constants. Define 


$$
\begin{aligned}
& V_{1}=x_{1}-x_{1}^{*}-x_{1}^{*} \ln \frac{x_{1}}{x_{1}^{*}}, \\
& V_{2}=x_{2}-x_{2}^{*}-x_{2}^{*} \ln \frac{x_{2}}{x_{2}^{*},} \\
& V_{3}=\left(u-u^{*}\right)^{2}, \\
& V_{4}=\frac{a_{12} f}{2 \omega_{2}} \int_{t-\tau_{2}}^{t}\left(x_{1}(s)-x_{1}^{*}\right)^{2} \mathrm{~d} s+\frac{a_{12} f \omega_{1}}{2} \int_{t-\tau_{1}}^{t}\left(x_{2}(s)-x_{2}^{*}\right)^{2} \mathrm{~d} s .
\end{aligned}
$$

By Itô's formula, we obtain

$$
\mathrm{d} V_{1}=L V_{1} \mathrm{~d} t+\left(x_{1}-x_{1}^{*}\right) \sigma_{1} \mathrm{~d} B_{1}(t),
$$

$$
\begin{aligned}
L V_{1} & =\left(x_{1}(t)-x_{1}^{*}\right)\left[r_{1}-a_{11} x_{1}(t)-a_{12} x_{2}\left(t-\tau_{1}\right)\right]+\frac{1}{2} x_{1}^{*} \sigma_{1}^{2} \\
& =\left(x_{1}(t)-x_{1}^{*}\right)\left[-a_{11}\left(x_{1}(t)-x_{1}^{*}\right)-a_{12}\left(x_{2}\left(t-\tau_{1}\right)-x_{2}^{*}\right)\right]+\frac{1}{2} x_{1}^{*} \sigma_{1}^{2} \\
& \leq-a_{11}\left(x_{1}(t)-x_{1}^{*}\right)^{2}+\frac{a_{12}}{2 \omega_{1}}\left(x_{1}(t)-x_{1}^{*}\right)^{2}+\frac{a_{12} \omega_{1}}{2}\left(x_{2}\left(t-\tau_{1}\right)-x_{2}^{*}\right)^{2}+\frac{1}{2} x_{1}^{*} \sigma_{1}^{2} .
\end{aligned}
$$

Similarly,

$$
d V_{2}=L V_{2} d t+\left(x_{2}-x_{2}^{*}\right) \sigma_{2} d B_{2}(t),
$$

$$
\begin{aligned}
L V_{2} & =\left(x_{2}(t)-x_{2}^{*}\right)\left[r_{2}+a_{21} x_{1}\left(t-\tau_{2}\right)-a_{22} x_{2}(t)-c u(t)\right]+\frac{1}{2} x_{2}^{*} \sigma_{2}^{2} \\
& =\left(x_{2}(t)-x_{2}^{*}\right)\left[a_{21}\left(x_{1}\left(t-\tau_{2}\right)-x_{1}^{*}\right)-a_{22}\left(x_{2}(t)-x_{2}^{*}\right)-c\left(u(t)-u^{*}\right)\right]+\frac{1}{2} x_{2}^{*} \sigma_{2}^{2} \\
& \leq \frac{a_{21}}{2 \omega_{2}}\left(x_{1}\left(t-\tau_{2}\right)-x_{1}^{*}\right)^{2}+\frac{a_{21} \omega_{2}}{2}\left(x_{2}(t)-x_{2}^{*}\right)^{2}-a_{22}\left(x_{2}(t)-x_{2}^{*}\right)^{2}-c\left(x_{2}(t)-x_{2}^{*}\right)\left(u(t)-u^{*}\right)+\frac{1}{2} x_{2}^{*} \sigma_{2}^{2} .
\end{aligned}
$$

In the same way,

$$
\begin{aligned}
\mathrm{d} V_{3} & =2\left(u(t)-u^{*}\right)\left(-e u(t)+f x_{2}(t)\right) \mathrm{d} t \\
& =\left[-2 e\left(u(t)-u^{*}\right)^{2}+2 f\left(u(t)-u^{*}\right)\left(x_{2}(t)-x_{2}^{*}\right)\right] \mathrm{d} t, \\
\mathrm{~d} V_{4} & =\left[\frac{a_{12} f}{2 \omega_{2}}\left(x_{1}(t)-x_{1}^{*}\right)^{2}-\frac{a_{12} f}{2 \omega_{2}}\left(x_{1}\left(t-\tau_{2}\right)-x_{1}^{*}\right)^{2}+\frac{a_{12} f \omega_{1}}{2}\left(x_{2}(t)-x_{2}^{*}\right)^{2}-\frac{a_{12} f \omega_{1}}{2}\left(x_{2}\left(t-\tau_{1}\right)-x_{2}^{*}\right)^{2}\right] \mathrm{d} t .
\end{aligned}
$$


Therefore, we have

$$
\begin{aligned}
L V= & f L V_{1}+\frac{a_{12} f}{a_{21}} L V_{2}+\frac{a_{12} c}{2 a_{21}} \frac{\mathrm{d} V_{3}}{\mathrm{~d} t}+\frac{\mathrm{d} V_{4}}{\mathrm{~d} t} \\
\leq & -a_{11} f\left(x_{1}(t)-x_{1}^{*}\right)^{2}+\frac{a_{12} f}{2 \omega_{1}}\left(x_{1}(t)-x_{1}^{*}\right)^{2}+\frac{a_{12} f \omega_{1}}{2}\left(x_{2}\left(t-\tau_{1}\right)-x_{2}^{*}\right)^{2}+\frac{1}{2} f x_{1}^{*} \sigma_{1}^{2} \\
& +\frac{a_{12} f}{2 \omega_{2}}\left(x_{1}\left(t-\tau_{2}\right)-x_{1}^{*}\right)^{2}+\frac{a_{12} f \omega_{2}}{2}\left(x_{2}(t)-x_{2}^{*}\right)^{2}-\frac{a_{12} a_{22} f}{a_{21}}\left(x_{2}(t)-x_{2}^{*}\right)^{2}-\frac{a_{12} c f}{a_{21}}\left(x_{2}(t)-x_{2}^{*}\right)\left(u(t)-u^{*}\right) \\
& +\frac{a_{12} f x_{2}^{*}}{2 a_{21}} \sigma_{2}^{2}-\frac{a_{12} c e}{a_{21}}\left(u(t)-u^{*}\right)^{2}+\frac{a_{12} c f}{a_{21}}\left(u(t)-u^{*}\right)\left(x_{2}(t)-x_{2}^{*}\right)+\frac{a_{12} f}{2 \omega_{2}}\left(x_{1}(t)-x_{1}^{*}\right)^{2}-\frac{a_{12} f}{2 \omega_{2}}\left(x_{1}\left(t-\tau_{2}\right)-x_{1}^{*}\right)^{2} \\
& +\frac{a_{12} f \omega_{1}}{2}\left(x_{2}(t)-x_{2}^{*}\right)^{2}-\frac{a_{12} f \omega_{1}}{2}\left(x_{2}\left(t-\tau_{1}\right)-x_{2}^{*}\right)^{2} \\
= & -f\left(a_{11}-\frac{a_{12}}{2 \omega_{1}}-\frac{a_{12}}{2 \omega_{2}}\right)\left(x_{1}(t)-x_{1}^{*}\right)^{2}-f\left(\frac{a_{12} a_{22}}{a_{21}}-\frac{a_{12} \omega_{2}}{2}-\frac{a_{12} \omega_{1}}{2}\right)\left(x_{2}(t)-x_{2}^{*}\right)^{2}-\frac{a_{12} c e}{a_{21}}\left(u(t)-u^{*}\right)^{2}+\frac{f x_{1}^{*}}{2} \sigma_{1}^{2} \\
& +\frac{a_{12} f x_{2}^{*}}{2 a_{21}} \sigma_{2}^{2} .
\end{aligned}
$$

Let $\omega_{1}=\omega_{2}=\left(\left(a_{11} a_{22}+a_{12} a_{21}\right) / 2 a_{11} a_{21}\right)$, we obtain

$$
\begin{aligned}
L V \leq & -\frac{a_{11} f\left(a_{11} a_{22}-a_{12} a_{21}\right)}{a_{11} a_{22}+a_{12} a_{21}}\left(x_{1}(t)-x_{1}^{*}\right)^{2}-\frac{a_{12} f\left(a_{11} a_{22}-a_{12} a_{21}\right)}{2 a_{11} a_{21}}\left(x_{2}(t)-x_{2}^{*}\right)^{2} \\
& -\frac{a_{12} c e}{a_{21}}\left(u(t)-u^{*}\right)^{2}+\frac{f x_{1}^{*}}{2} \sigma_{1}^{2}+\frac{a_{12} f x_{2}^{*}}{2 a_{21}} \sigma_{2}^{2} .
\end{aligned}
$$

Therefore,

$$
\begin{aligned}
\mathrm{d} V\left(x_{1}, x_{2}, u\right)= & L V \mathrm{~d} t+f\left(x_{1}-x_{1}^{*}\right) \sigma_{1} \mathrm{~d} B_{1}(t) \\
& +\frac{a_{12} f}{a_{21}}\left(x_{2}-x_{2}^{*}\right) \sigma_{2} \mathrm{~d} B_{2}(t) .
\end{aligned}
$$

$$
\begin{aligned}
E V(t)-E V(0) \leq & -E \int_{0}^{t} \frac{a_{11} f\left(a_{11} a_{22}-a_{12} a_{21}\right)}{a_{11} a_{22}+a_{12} a_{21}}\left(x_{1}(\theta)-x_{1}^{*}\right)^{2} \mathrm{~d} \theta \\
& -E \int_{0}^{t} \frac{a_{12} f\left(a_{11} a_{22}-a_{12} a_{21}\right)}{2 a_{11} a_{21}}\left(x_{2}(\theta)-x_{2}^{*}\right)^{2} \mathrm{~d} \theta-E \int_{0}^{t} \frac{a_{12} c e}{a_{21}}\left(u(\theta)-u^{*}\right)^{2} \mathrm{~d} \theta+\frac{f x_{1}^{*} \sigma_{1}^{2} t}{2}+\frac{a_{12} f x_{2}^{*} \sigma_{2}^{2} t}{2 a_{21}}
\end{aligned}
$$

Divide both sides by $t$ and take the limit superior, and then we have 


$$
\begin{aligned}
& \underset{t \rightarrow+\infty}{\limsup } \frac{1}{t} E \int_{0}^{t} \frac{a_{11} f\left(a_{11} a_{22}-a_{12} a_{21}\right)}{a_{11} a_{22}+a_{12} a_{21}}\left(x_{1}(\theta)-x_{1}^{*}\right)^{2} \mathrm{~d} \theta \\
& \quad+\limsup _{t \longrightarrow+\infty} \frac{1}{t} E \int_{0}^{t} \frac{a_{12} f\left(a_{11} a_{22}-a_{12} a_{21}\right)}{2 a_{11} a_{21}}\left(x_{2}(\theta)-x_{2}^{*}\right)^{2} \mathrm{~d} \theta \\
& \quad+\limsup _{t \longrightarrow+\infty} \frac{1}{t} E \int_{0}^{t} \frac{a_{12} c e}{a_{21}}\left(u(\theta)-u^{*}\right)^{2} \mathrm{~d} \theta \leq \frac{f x_{1}^{*} \sigma_{1}^{2}}{2}+\frac{a_{12} f x_{2}^{*} \sigma_{2}^{2}}{2 a_{21}} .
\end{aligned}
$$

Obviously,

$$
\underset{t \longrightarrow+\infty}{\limsup } \frac{1}{t} E \int_{0}^{t}\left[\left(x_{1}(\theta)-x_{1}^{*}\right)^{2}+\left(x_{2}(\theta)-x_{2}^{*}\right)^{2}+\left(u(\theta)-u^{*}\right)^{2}\right] \mathrm{d} \theta \leq \frac{n}{m}
$$

where

$$
\begin{aligned}
& m=\min \left\{\frac{a_{11} f\left(a_{11} a_{22}-a_{12} a_{21}\right)}{a_{11} a_{22}+a_{12} a_{21}}, \frac{a_{12} f\left(a_{11} a_{22}-a_{12} a_{21}\right)}{2 a_{11} a_{21}}, \frac{a_{12} c e}{a_{21}}\right\}, \\
& n=\frac{f x_{1}^{*} \sigma_{1}^{2}}{2}+\frac{a_{12} f x_{2}^{*} \sigma_{2}^{2}}{2 a_{21}} .
\end{aligned}
$$

We have completed the proof.

Theorem 2 shows that if the condition $\left(A_{1}\right)$ holds, the solution oscillates around the equilibrium point $x^{*}$, and the amplitude of oscillation is positively correlated with the intensity $\sigma_{1}^{2}$ and $\sigma_{2}^{2}$ of environmental noise. In particular, if $\sigma_{1}^{2}=\sigma_{2}^{2}=0$, the influence of environmental noise is not taken into account:

$x_{1}(t) \longrightarrow x_{1}^{*}, x_{2}(t) \longrightarrow x_{2}^{*}, u(t) \longrightarrow u^{*}$ for $t \longrightarrow+\infty$.
The equilibrium point $x^{*}$ is globally asymptotically stable. This is the conclusion of reference [17].

\section{Persistence}

In nature, whether ecosystems can survive or not is our main concern. Before discussing the persistence of stochastic system, we give the following assumption:

$$
\left(A_{2}\right): \mu=\max \left(\sigma_{1}, \sigma_{2}\right)<\min \left\{x_{1}^{*} \sqrt{\frac{m}{n_{0}}}, x_{2}^{*} \sqrt{\frac{m}{n_{0}}}, u^{*} \sqrt{\frac{m}{n_{0}}}\right\}, \quad n_{0}=\frac{f x_{1}^{*}}{2}+\frac{a_{12} f x_{2}^{*}}{2 a_{21}} .
$$

Theorem 3. For any given initial condition (6), if assumptions $\left(A_{1}\right)$ and $\left(A_{2}\right)$ hold at the same time, the solution $\left(x_{1}(t), x_{2}(t), u(t)\right)$ of system (5) is persistent that

$$
\begin{aligned}
& \liminf _{t \rightarrow \infty} \frac{1}{t} E \int_{0}^{t} x_{1}(\theta) \mathrm{d} \theta>0, \\
& \liminf _{t \longrightarrow \infty} \frac{1}{t} E \int_{0}^{t} x_{2}(\theta) \mathrm{d} \theta>0, \\
& \liminf _{t \rightarrow \infty} \frac{1}{t} E \int_{0}^{t} u(\theta) \mathrm{d} \theta>0 .
\end{aligned}
$$

Proof. According to (37), we have

$$
\begin{aligned}
& \limsup _{t \longrightarrow+\infty} \frac{1}{t} E \int_{0}^{t}\left[\left(x_{1}(\theta)-x_{1}^{*}\right)^{2}\right] \mathrm{d} \theta \leq \frac{n}{m}, \\
& \underset{t \rightarrow+\infty}{\limsup } \frac{1}{t} E \int_{0}^{t}\left[\left(x_{2}(\theta)-x_{2}^{*}\right)^{2}\right] \mathrm{d} \theta \leq \frac{n}{m}, \\
& \underset{t \rightarrow+\infty}{\limsup } \frac{1}{t} E \int_{0}^{t}\left[\left(u(\theta)-u^{*}\right)^{2}\right] \mathrm{d} \theta \leq \frac{n}{m} .
\end{aligned}
$$

As we know, $x_{1}(t) \geq 0$ and $x_{1}^{*}>0$, from $2 x_{1}(t) x_{1}^{*}$ $\geq\left(x_{1}^{*}\right)^{2}-\left(x_{1}(t)-x_{1}^{*}\right)^{2}$, one can get 


$$
x_{1}(t) \geq \frac{x_{1}^{*}}{2}-\frac{\left(x_{1}(t)-x_{1}^{*}\right)^{2}}{2 x_{1}^{*}} .
$$

By the condition $\mu<x_{1}^{*} \sqrt{m / n_{0}}$, we have

$$
\begin{aligned}
& \liminf _{t \rightarrow \infty} \frac{1}{t} E \int_{0}^{t} x_{1}(\theta) \mathrm{d} \theta \\
& \geq \frac{x_{1}^{*}}{2}-\limsup _{t \longrightarrow \infty} \frac{1}{t} E \int_{0}^{t} \frac{\left(x_{1}(\theta)-x_{1}^{*}\right)^{2}}{2 x_{1}^{*}} \mathrm{~d} \theta \\
& \geq \frac{x_{1}^{*}}{2}-\frac{n}{2 m x_{1}^{*}} \geq \frac{x_{1}^{*}}{2}-\frac{\mu^{2} n_{0}}{2 m x_{1}^{*}}>0 .
\end{aligned}
$$

Similarly, when $\mu<\min \left\{x_{2}^{*} \sqrt{m / n_{0}}, u^{*} \sqrt{m / n_{0}}\right\}$,

$$
\begin{aligned}
& \underset{t \rightarrow \infty}{\liminf } \frac{1}{t} E \int_{0}^{t} x_{2}(\theta) \mathrm{d} \theta>0 \\
& \liminf _{t \rightarrow \infty} \frac{1}{t} E \int_{0}^{t} u(\theta) \mathrm{d} \theta>0 .
\end{aligned}
$$

\section{Extinction}

Define

$$
\begin{aligned}
\Delta= & a_{21}\left(r_{1}-\frac{1}{2} \sigma_{1}^{2}\right)+a_{11}\left(r_{2}-\frac{1}{2} \sigma_{2}^{2}\right), \\
\Delta_{1}= & e\left(a_{11} a_{22}+a_{12} a_{21}\right)-a_{11} c f, \\
\Delta_{2}= & a_{12} \Delta\left[e\left(a_{11} a_{22}+a_{12} a_{21}\right)-a_{11} c f\right] \\
& -\left(r_{1}-\frac{1}{2} \sigma_{1}^{2}\right) e\left(a_{11} a_{22}+a_{12} a_{21}\right)^{2} .
\end{aligned}
$$

For the extinction of system (5), we have the following conclusions.

Theorem 4. For any given initial condition (6), the solution $\left(x_{1}(t), x_{2}(t), u(t)\right)$ of system (5) has the following properties:

(i) when $r_{1}-(1 / 2) \sigma_{1}^{2}<0$ and $r_{2}-(1 / 2) \sigma_{2}^{2}<0$, the population is extinct

(ii) when $r_{1}-(1 / 2) \sigma_{1}^{2}>0$, and $\Delta<0$, population $x_{1}$ is persistent and $x_{2}$ and $u$ are extinct

(iii) when $r_{1}-(1 / 2) \sigma_{1}^{2}>0, \Delta>0, \Delta_{1}>0$, and $\Delta_{2}>0$, population $x_{1}$ is extinct and $x_{2}$ and $u$ are persistent

Before proving Theorem 4, consider the following auxiliary system [37]:

$$
\left\{\begin{array}{l}
\mathrm{d} z_{1}(t)=z_{1}(t)\left[r_{1}-a_{11} z_{1}(t)\right] \mathrm{d} t+\sigma_{1} z_{1}(t) \mathrm{d} B_{1}(t) \\
\mathrm{d} z_{2}(t)=z_{2}(t)\left[r_{2}+a_{21} z_{1}\left(t-\tau_{2}\right)-a_{22} z_{2}(t)\right] \mathrm{d} t \\
\quad+\sigma_{2} z_{2}(t) \mathrm{d} B_{2}(t)
\end{array}\right.
$$

and it satisfies the initial condition: $\phi(\theta)=\left(\phi_{1}(\theta)\right.$, $\left.\phi_{2}(\theta)\right) \in C\left([-\tau, 0], R_{+}^{2}\right)$.

Lemma 1. If $r_{1}-(1 / 2) \sigma_{1}^{2}>0$, the solution $z(t)$ of system (47) has the following properties:

(i) $\lim _{t \longrightarrow+\infty} t^{-1} \int_{0}^{t} z_{1}(s) d s=\left(r_{1}-\left((1 / 2) \sigma_{1}^{2}\right)\right) / a_{11}$

(ii) if $\Delta<0, \lim _{t \rightarrow+\infty} z_{2}(t)=0$

(iii) if $\Delta>0, \lim _{t \longrightarrow+\infty} t^{-1} \int_{0}^{t} z_{2}(s) \mathrm{d} s=\left(\Delta / a_{11} a_{22}\right)$, a.s

Proof. By Itô's formula, we obtain

$$
\begin{aligned}
\ln z_{1}(t)-\ln z_{1}(0) & =\left(r_{1}-\frac{1}{2} \sigma_{1}^{2}\right) t-a_{11} \int_{0}^{t} z_{1}(s) \mathrm{d} s+\sigma_{1} B_{1}(\mathrm{t}) \\
\ln z_{2}(t)-\ln z_{2}(0) & =\left(r_{2}-\frac{1}{2} \sigma_{2}^{2}\right) t+a_{21} \int_{0}^{t} z_{1}\left(s-\tau_{2}\right) \mathrm{d} s-a_{22} \int_{0}^{t} z_{2}(s) \mathrm{d} s+\sigma_{2} B_{2}(t) \\
& =\left(r_{2}-\frac{1}{2} \sigma_{2}^{2}\right) t+a_{21} \int_{0}^{t} z_{1}(s) \mathrm{d} s-a_{21}\left[\int_{t-\tau_{2}}^{t} z_{1}(s) \mathrm{d} s-\int_{-\tau_{2}}^{0} z_{1}(s) \mathrm{d} s\right]-a_{22} \int_{0}^{t} z_{2}(s) \mathrm{d} s+\sigma_{2} B_{2}(t)
\end{aligned}
$$

Dividing both sides of (48) and (49) by $t$, we have

$$
\begin{gathered}
t^{-1} \ln \frac{z_{1}(t)}{z_{1}(0)}=\left(r_{1}-\frac{1}{2} \sigma_{1}^{2}\right)-a_{11} t^{-1} \int_{0}^{t} z_{1}(s) \mathrm{d} s+t^{-1} \sigma_{1} B_{1}(t) \\
t^{-1} \ln \frac{z_{2}(t)}{z_{2}(0)}+t^{-1} a_{21}\left[\int_{t-\tau_{2}}^{t} z_{1}(s) \mathrm{d} s-\int_{-\tau_{2}}^{0} z_{1}(s) \mathrm{d} s\right]=\left(r_{2}-\frac{1}{2} \sigma_{2}^{2}\right)+a_{21} t^{-1} \int_{0}^{t} z_{1}(s) \mathrm{d} s-a_{22} t^{-1} \int_{0}^{t} z_{2}(s) \mathrm{d} s+t^{-1} \sigma_{2} B_{2}(t)
\end{gathered}
$$



that

By using Lemma 2 of [37], from equation (48), it follows

$$
\begin{aligned}
& \limsup _{t \longrightarrow+\infty} t^{-1} \int_{0}^{t} z_{1}(s) \mathrm{d} s \leq \frac{r_{1}-(1 / 2) \sigma_{1}^{2}}{a_{11}} \\
& \liminf _{t \longrightarrow+\infty} t^{-1} \int_{0}^{t} z_{1}(s) \mathrm{d} s \geq \frac{r_{1}-(1 / 2) \sigma_{1}^{2}}{a_{11}},
\end{aligned}
$$

and therefore,

$$
\lim _{t \longrightarrow+\infty} t^{-1} \int_{0}^{t} z_{1}(s) \mathrm{d} s=\frac{r_{1}-(1 / 2) \sigma_{1}^{2}}{a_{11}} .
$$

Substitute (53) into (50), and from $\lim _{t \longrightarrow+\infty} t^{-1} B_{i}(t)=0$ $(i=1,2)$, we get

$$
\lim _{t \longrightarrow+\infty} t^{-1} \ln z_{1}(t)=0
$$

On the other hand, computing $(50) \times a_{21}+(51) \times a_{11}$, we have

$$
\begin{aligned}
& a_{21} t^{-1} \ln \frac{z_{1}(t)}{z_{1}(0)}+a_{11} t^{-1} \ln \frac{z_{2}(t)}{z_{2}(0)}+t^{-1} a_{11} a_{21}\left[\int_{t-\tau_{2}}^{t} z_{1}(s) \mathrm{d} s-\int_{-\tau_{2}}^{0} z_{1}(s) \mathrm{d} s\right] \\
& \quad=a_{21}\left(r_{1}-\frac{1}{2} \sigma_{1}^{2}\right)+a_{11}\left(r_{2}-\frac{1}{2} \sigma_{2}^{2}\right)-a_{11} a_{22} t^{-1} \int_{0}^{t} z_{2}(s) \mathrm{d} s+t^{-1}\left[a_{21} \sigma_{1} B_{1}(t)+a_{11} \sigma_{2} B_{2}(t)\right] .
\end{aligned}
$$

By Lemma 2 in literature [37], when $\Delta<0$,

Proof of lemma is completed.

$$
\lim _{t \rightarrow+\infty} z_{2}(t)=0
$$

If $\Delta>0$,

$$
\lim _{t \longrightarrow+\infty} t^{-1} \int_{0}^{t} z_{2}(s) \mathrm{d} s=\frac{\Delta}{a_{11} a_{22}} \text { a.s. }
$$

This is where we prove Theorem 4 .

Proof. By using Itô's formula for system (5), we have

$$
\begin{aligned}
\ln x_{1}(t)-\ln x_{1}(0)= & \left(r_{1}-\frac{1}{2} \sigma_{1}^{2}\right) t-a_{11} \int_{0}^{t} x_{1}(s) \mathrm{d} s-a_{12} \int_{0}^{t} x_{2}\left(s-\tau_{1}\right) \mathrm{d} s+\sigma_{1} B_{1}(t) \\
= & \left(r_{1}-\frac{1}{2} \sigma_{1}^{2}\right) t-a_{11} \int_{0}^{t} x_{1}(s) \mathrm{d} s-a_{12} \int_{0}^{t} x_{2}(s) \mathrm{d} s+a_{12}\left[\int_{t-\tau_{1}}^{t} x_{2}(s) \mathrm{d} s-\int_{-\tau_{1}}^{0} x_{2}(s) \mathrm{d} s\right]+\sigma_{1} B_{1}(t), \\
\ln x_{2}(t)-\ln x_{2}(0)= & \left(r_{2}-\frac{1}{2} \sigma_{2}^{2}\right) t+a_{21} \int_{0}^{t} x_{1}\left(s-\tau_{2}\right) \mathrm{d} s-a_{22} \int_{0}^{t} x_{2}(s) \mathrm{d} s-c \int_{0}^{t} u(s) \mathrm{d} s+\sigma_{2} B_{2}(t) \\
= & \left(r_{2}-\frac{1}{2} \sigma_{2}^{2}\right) t+a_{21} \int_{0}^{t} x_{1}(s) \mathrm{d} s-a_{21}\left[\int_{t-\tau_{2}}^{t} x_{1}(s) \mathrm{d} s-\int_{-\tau_{2}}^{0} x_{1}(s) \mathrm{d} s\right] \\
& -a_{22} \int_{0}^{t} x_{2}(s) \mathrm{d} s-c \int_{0}^{t} u(s) \mathrm{d} s+\sigma_{2} B_{2}(t) .
\end{aligned}
$$

We first prove (i): from equation (58), we can get $\ln x_{1}(t)-\ln x_{1}(0) \leq\left(r_{1}-\frac{1}{2} \sigma_{1}^{2}\right) t-a_{11} \int_{0}^{t} x_{1}(s) \mathrm{d} s+\sigma_{1} B_{1}(t)$.

By the condition $r_{1}-(1 / 2) \sigma_{1}^{2}<0$ and Lemma 2 in literature [37],

$$
\lim _{t \longrightarrow+\infty} x_{1}(t)=0 \text { a.s. }
$$

From (57), (61), and the condition $r_{2}-(1 / 2) \sigma_{2}^{2}<0$, we have

$$
\lim _{t \longrightarrow+\infty} x_{2}(t)=0 \text { a.s. }
$$

Further, from the third equation of model (5), we can get

$$
\lim _{t \rightarrow+\infty} u(t)=0 \text { a.s. }
$$

Secondly, it proves (ii): comparing model (5) with auxiliary system (37), one can get $x_{1}(t) \leq z_{1}(t)$ and $x_{2}(t) \leq z_{2}(t)$. By Lemma 1 , when $r_{1}-(1 / 2) \sigma_{1}^{2}>0$ and $\Delta<0$, $\lim _{t \longrightarrow+\infty} z_{2}(t)=0$ a.s.; therefore, $\lim _{t \longrightarrow+\infty} x_{2}(t)=0$ a.s. So, we have $\lim _{t \rightarrow+\infty} u(t)=0$. Then, the limit system of model (5) is as follows:

$$
\mathrm{d} x_{1}(t)=x_{1}(t)\left[r_{1}-a_{11} x_{1}(t)\right] \mathrm{d} t+\sigma_{1} x_{1}(t) \mathrm{d} B_{1}(t) .
$$

From Lemma 1, we can conclude that 


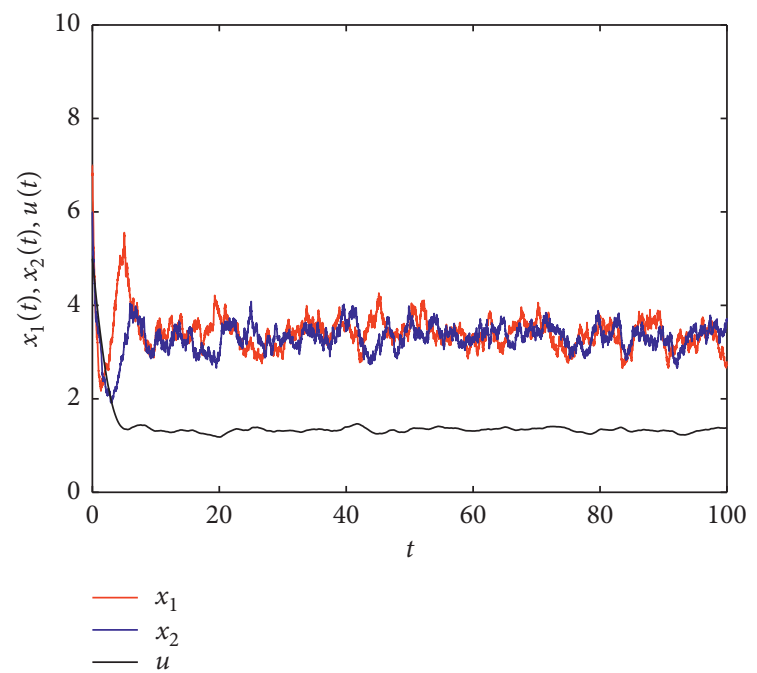

(a)

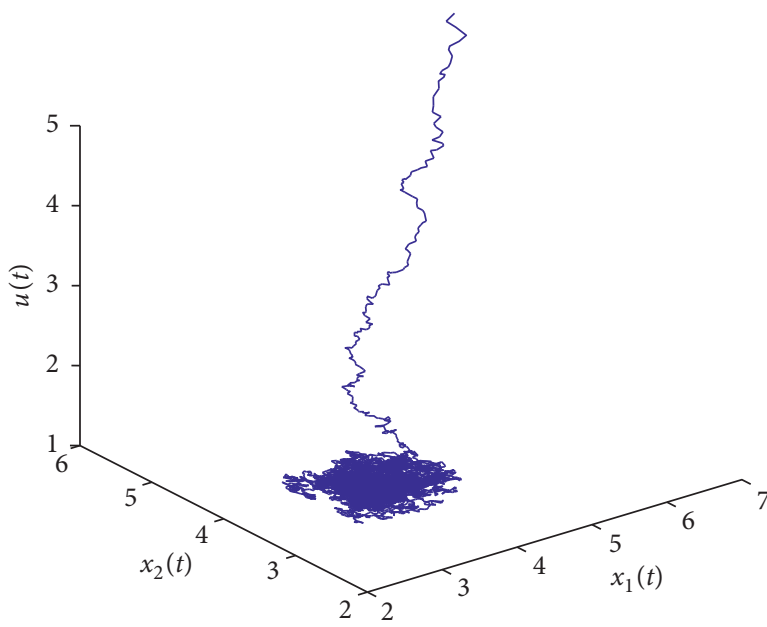

(b)

Figure 1: (a) Time series diagram of $\left(x_{1}(t), x_{2}(t), u(t)\right)$ and (b) its phase diagram.

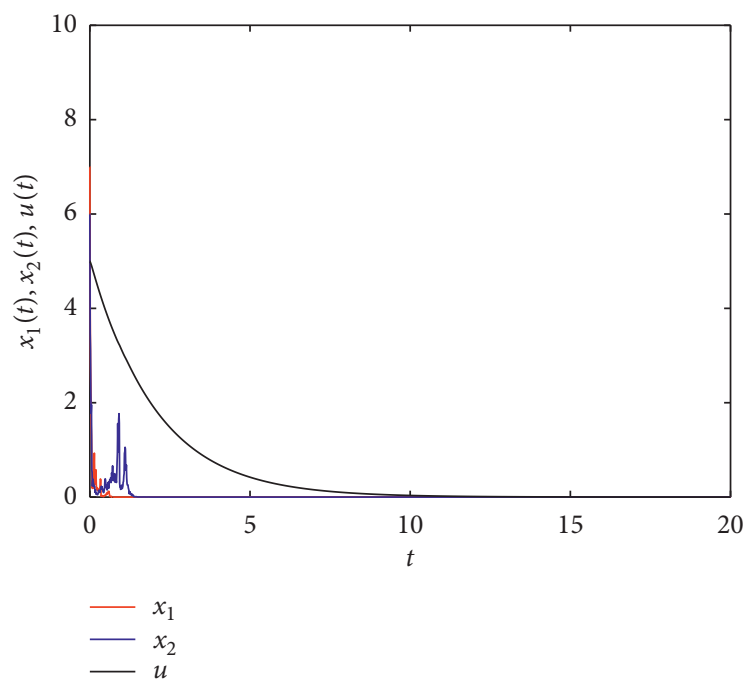

Figure 2: Time series diagram of $\left(x_{1}(t), x_{2}(t), u(t)\right)$.

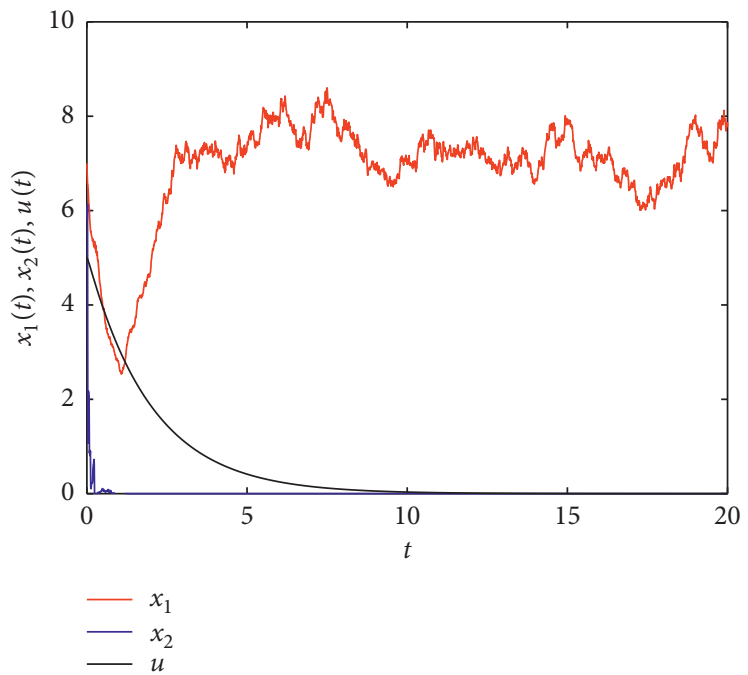

Figure 3: Time series diagram of $\left(x_{1}(t), x_{2}(t), u(t)\right)$. 


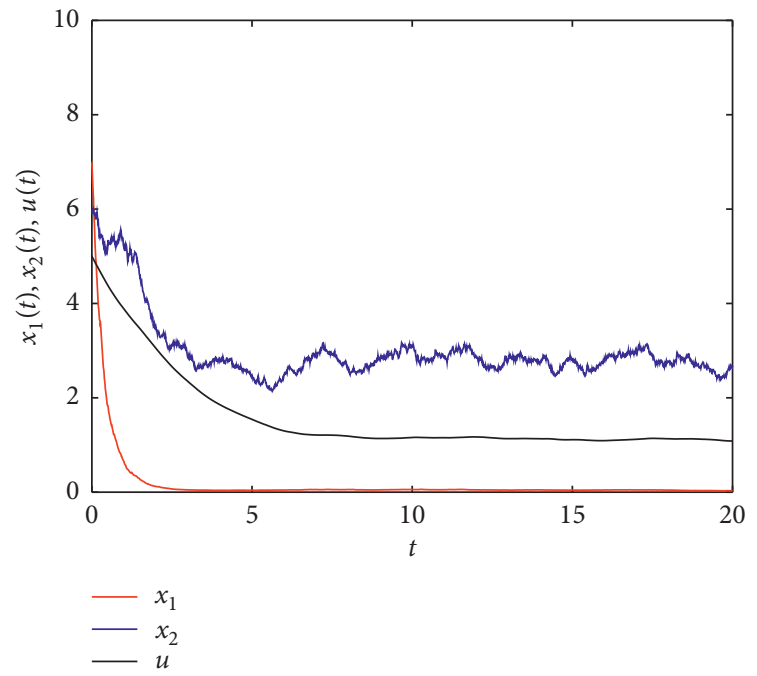

(a)

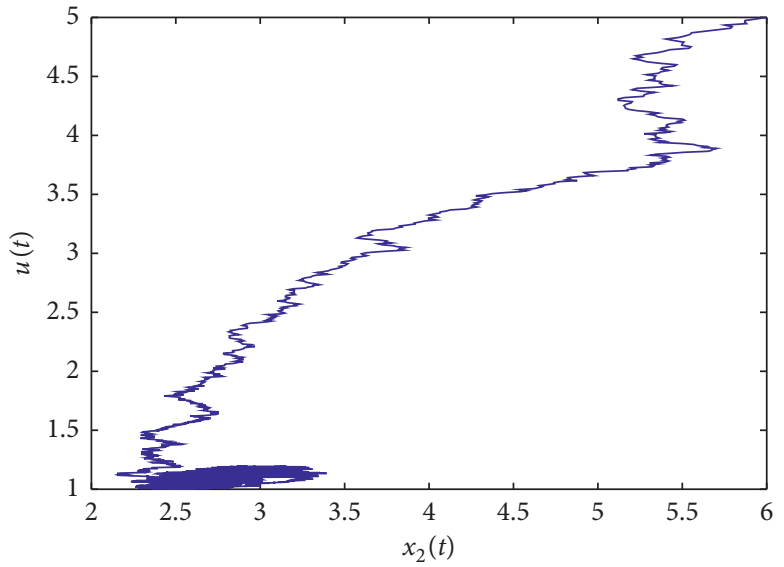

(b)

Figure 4: (a) Time series diagram of $\left(x_{1}(t), x_{2}(t), u(t)\right)$. (b) Phase diagram of $\left(x_{2}(t)\right), u(t)$.

$$
\lim _{t \longrightarrow+\infty} t^{-1} \int_{0}^{t} x_{1}(s) \mathrm{d} s=\frac{r_{1}-(1 / 2) \sigma_{1}^{2}}{a_{11}} \text { a.s. }
$$

Therefore, population $x_{1}$ is persistent and $x_{2}$ and $u$ are extinct.

$$
\begin{gathered}
\limsup _{t \longrightarrow+\infty} t^{-1} \ln x_{1}(t) \leq \lim _{t \rightarrow+\infty} t^{-1} \ln z_{1}(t)=0 . \\
\text { Computing }(58) \times a_{21}+(59) \times a_{11},
\end{gathered}
$$

Therefore,

$$
\begin{aligned}
& a_{21} t^{-1} \ln \frac{x_{1}(t)}{x_{1}(0)}+a_{11} t^{-1} \ln \frac{x_{2}(t)}{x_{2}(0)}+t^{-1} a_{11} a_{21}\left[\int_{t-\tau_{2}}^{t} x_{1}(s) \mathrm{d} s-\int_{-\tau_{2}}^{0} x_{1}(s) \mathrm{d} s\right]-t^{-1} a_{12} a_{21}\left[\int_{t-\tau_{1}}^{t} x_{2}(s) \mathrm{d} s-\int_{-\tau_{1}}^{0} x_{2}(s) \mathrm{d} s\right] \\
& \leq a_{21}\left(r_{1}-\frac{1}{2} \sigma_{1}^{2}\right)+a_{11}\left(r_{2}-\frac{1}{2} \sigma_{2}^{2}\right)-\left(a_{11} a_{22}+a_{12} a_{21}\right) t^{-1} \int_{0}^{t} x_{2}(s) \mathrm{d} s+t^{-1}\left[a_{21} \sigma_{1} B_{1}(t)+a_{11} \sigma_{2} B_{2}(t)\right] .
\end{aligned}
$$

Let $t \longrightarrow+\infty$ and the condition $\Delta=a_{21}\left(r_{1}-(1)\right.$ 2) $\left.\sigma_{1}^{2}\right)+a_{11}\left(r_{2}-(1 / 2) \sigma_{2}^{2}\right)>0$ is satisfied, so

$$
\limsup _{t \longrightarrow+\infty} t^{-1} \int_{0}^{t} x_{2}(s) \mathrm{d} s \leq \frac{\Delta}{a_{11} a_{22}+a_{12} a_{21}} \text { a.s. }
$$

Considering the third equation of model (5),

$$
\frac{u(t)-u(0)}{t}=-e t^{-1} \int_{0}^{t} u(s) \mathrm{d} s+f t^{-1} \int_{0}^{t} x_{2}(s) \mathrm{d} s
$$

hence, 


$$
\limsup _{t \longrightarrow+\infty} t^{-1} \int_{0}^{t} u(s) \mathrm{d} s \leq \frac{f \Delta}{e\left(a_{11} a_{22}+a_{12} a_{21}\right)} \text { a.s. }
$$

Substitute (71) into equation (67), and by the condition $\Delta_{1}=e\left(a_{11} a_{22}+a_{12} a_{21}\right)-a_{11} c f>0$, we have

$$
\begin{gathered}
\liminf _{t \longrightarrow+\infty} t^{-1} \int_{0}^{t} x_{2}(s) \mathrm{d} s \geq \frac{\Delta-\left(a_{11} c f \Delta / e\left(a_{11} a_{22}+a_{12} a_{21}\right)\right)}{a_{11} a_{22}+a_{12} a_{21}} \\
=\frac{\left(e\left(a_{11} a_{22}+a_{12} a_{21}\right)-a_{11} c f\right) \Delta}{e\left(a_{11} a_{22}+a_{12} a_{21}\right)^{2}}>0 \text { a.s. }
\end{gathered}
$$

It is concluded that predator $x_{2}(t)$ is persistent.

When $t \longrightarrow+\infty$ and substituting (72) into equation (70), one can see that

$$
\liminf _{t \longrightarrow+\infty} t^{-1} \int_{0}^{t} u(s) \mathrm{d} s \geq \frac{f\left[e\left(a_{11} a_{22}+a_{12} a_{21}\right)-a_{11} c f\right] \Delta}{e^{2}\left(a_{11} a_{22}+a_{12} a_{21}\right)^{2}}>0 \text { a.s. }
$$

So, $u(t)$ is persistent.

The condition $\Delta_{2}>0$ means that

$$
r_{1}-\frac{1}{2} \sigma_{1}^{2}<\frac{a_{12}\left[e\left(a_{11} a_{22}+a_{12} a_{21}\right)-a_{11} c f\right] \Delta}{e\left(a_{11} a_{22}+a_{12} a_{21}\right)^{2}} .
$$

Substituting (72) into equation (58) and by the condition $\Delta_{2}>0$, we have

$$
\lim _{t \longrightarrow+\infty} x_{1}(t)=0 \text { a.s. }
$$

So, $x_{1}(t)$ is extinction. The proof of Theorem 4 is complete.

\section{Conclusions and Numerical Simulations}

This paper proposes a stochastic Lotka-Volterra predatorprey model with discrete delays and feedback control. We firstly study the existence and uniqueness of global positive solution. By constructing appropriate Lyapunov functions and applying Itô's formula, we discuss the asymptotic behavior of stochastic system at the positive equilibrium point of the corresponding deterministic model. Finally, this paper gives the conditions for the persistence and extinction of stochastic system. Theorem 3 shows that the system is persistent if the intensity $\sigma_{i}(i=1,2)$ of random disturbance and the coefficient $c$ of feedback control variable satisfy the condition $\left(A_{2}\right)$. Theorem 4 indicates that

(1) If the coefficient $c$ of the feedback control $c u(t) x_{2}(t)$ remains unchanged and the intensity $\sigma_{i}(i=1,2)$ of random disturbance increases, the population cannot resist the disturbance of the external environment and extinct

(2) If the intensity of random disturbance $\sigma_{i}(i=1,2)$ remains unchanged and the coefficient $c$ of feedback control $c u(t) x_{2}(t)$ is small, $\Delta_{1}>0$ and $\Delta_{2}>0$ are satisfied, which will cause the continuous increase of predator number for a period of time, thus leading to the extinction of the prey population.

Therefore, utilizing the feedback control measures to limit the predator quantity within a certain range is beneficial for the sustained existence of the population.

In order to verify the correctness of the theoretical analysis, we carry out the following numerical simulations. Choose the parameters in system (5) as follows:

$$
\begin{aligned}
r_{1} & =1.5, \\
r_{2} & =1.2, \\
e & =0.5, \\
f & =0.2, \\
a_{11} & =0.2, \\
a_{21} & =0.2, \\
a_{22} & =0.4, \\
\tau_{1} & =1, \\
\tau_{2} & =1 .
\end{aligned}
$$

Let $\Delta t=0.01$ and the initial value $x_{1}(0)=$ $7, x_{2}(0)=6$, and $u(0)=5$.

(i) Set $c=0.4, a_{12}=0.25, \sigma_{1}=0.05$, and $\sigma_{2}=0.05$, and the positive equilibrium point of the corresponding deterministic model is $\left(x_{1}^{*}, x_{2}^{*}, u^{*}\right)=(10 / 3,10 /$ $3,4 / 3)$. It is proved that conditions $\left(A_{1}\right)$ and $\left(A_{2}\right)$ are satisfied and Theorem 2 and Theorem 3 are valid. System (5) oscillates slightly near the point $(10 / 3,10 / 3,4 / 3)$ and persistently (see Figure 1$)$.

(ii) Set $c=0.1, a_{12}=0.25, \sigma_{1}=1.8$, and $\sigma_{2}=1.6$, one can get $r_{1}-(1 / 2) \sigma_{1}^{2}=-0.12<0$ and $r_{2}-(1 / 2) \sigma_{2}^{2}=$ $-0.08<0$. According to Theorem 4 (i), system (5) is extinct (see Figure 2).

(iii) Set $c=0.1, a_{12}=0.25, \sigma_{1}=0.05$, and $\sigma_{2}=2.5$, we have $r_{1}-(1 / 2) \sigma_{1}^{2}=1.4988>0$ and $\Delta=a_{21}\left(r_{1}-\right.$ $\left.(1 / 2) \sigma_{1}^{2}\right)+a_{11}\left(r_{2}-(1 / 2) \sigma_{2}^{2}\right)=-0.0852<0$.

According to Theorem 4 (ii), population $x_{1}$ is persistent and $x_{2}$ and $u$ are extinct (see Figure 3 ).

(iv) Set $c=0.1, a_{12}=0.55, \sigma_{1}=0.05$ and $\sigma_{2}=0.05$, we obtain $r_{1}-(1 / 2) \sigma_{1}^{2}=1.4988>0, \Delta=a_{21}\left(r_{1}-(1 / 2)\right.$ $\left.\sigma_{1}^{2}\right)+a_{11}\left(r_{2}-(1 / 2) \sigma_{2}^{2}\right)=0.5395>0, \Delta_{1}=e\left(a_{11} a_{22}\right.$ $\left.+a_{12} a_{21}\right)-a_{11} c f=0.091>0$, and $\Delta_{2}=a_{12} \Delta\left[e\left(a_{11}\right.\right.$ $\left.\left.a_{22}+a_{12} a_{21}\right)-a_{11} c f\right]-\left(r_{1}-(1 / 2) \sigma_{1}^{2}\right) e\left(a_{11} a_{22}+a_{12}\right.$ $\left.a_{21}\right)^{2}=0.027>0$. According to Theorem 4 (iii), population $x_{1}$ is extinct and $x_{2}$ and $u$ are persistent (see Figure 4 ).

\section{Data Availability}

All data sets used in this study are hypothetical.

\section{Conflicts of Interest}

The authors declare that they have no conflicts of interest. 


\section{Acknowledgments}

This work was supported by the National Natural Science Foundation of China (11371230) and SDUST Research Fund (2014TDJH102).

\section{References}

[1] Y. Muroya, "Permanence and global stability in a lotka-volterra predator-prey system with delays," Applied Mathematics Letters, vol. 16, no. 8, pp. 1245-1250, 2003.

[2] X. Meng, F. Li, and S. Gao, "Global analysis and numerical simulations of a novel stochastic eco-epidemiological model with time delay," Applied Mathematics and Computation, vol. 339, pp. 701-726, 2018.

[3] T. Ma, X. Meng, and Z. Chang, "Dynamics and optimal harvesting control for a stochastic one-predator-two-prey time delay system with jumps," Complexity, vol. 2019, Article ID 5342031, p. 19, 2019.

[4] Z. Jiang, X. Bi, T. Zhang, and B. S. A. Pradeep, "Global hopf bifurcation of a delayed phytoplankton-zooplankton system considering toxin producing effect and delay dependent coefficient," Mathematical Biosciences and Engineering, vol. 16, no. 5, pp. 3807-3829, 2019.

[5] F. Li, S. Zhang, and X. Meng, "Dynamics analysis and numerical simulations of a delayed stochastic epidemic model subject to a general response function," Computational and Applied Mathematics, vol. 38, no. 2, p. 95, 2019.

[6] Y. Saito, T. Hara, and W. Ma, "Necessary and sufficient conditions for permanence and global stability of a lotkavolterra system with two delays," Journal of Mathematical Analysis and Applications, vol. 236, no. 2, pp. 534-556, 1999.

[7] H. Hu, Z. Teng, and S. Gao, "Extinction in nonautonomous lotka-volterra competitive system with pure-delays and feedback controls," Nonlinear Analysis: Real World Applications, vol. 10, no. 4, pp. 2508-2520, 2009.

[8] H. Huo and W. Li, "Positive periodic solutions of a class of delay differential system with feedback control," Applied Mathematics and Computation, vol. 148, no. 1, pp. 35-46, 2004.

[9] W. Wang, W. Ma, and Z. Feng, "Dynamics of reactiondiffusion equations for modeling $\mathrm{CD} 4^{+} \mathrm{T}$ cells decline with general infection mechanism and distinct dispersal rates," Nonlinear Analysis: Real World Applications, vol. 51, Article ID 102976, 2020.

[10] W. Lv, F. Wang, and Y. Li, "Adaptive finite-time tracking control for nonlinear systems with unmodeled dynamics using neural networks," Advances in Difference Equations, vol. 2018, Article ID 159, 2018.

[11] J. Wang, H. Cheng, H. Liu, and Y. Wang, "Periodic solution and control optimization of a prey-predator model with two types of harvesting," Advances in Difference Equations, vol. 2018, Article ID 41, 2018.

[12] W. Lv and F. Wang, "Adaptive tracking control for a class of uncertain nonlinear systems with infinite number of actuator failures using neural networks," Advances in Difference Equations, vol. 2017, no. 1, Article ID 374, 2017.

[13] T. Zhang, N. Gao, T. Wang, H. Liu, and Z. Jiang, "Global dynamics of a model for treating microorganisms in sewage by periodically adding microbial flocculants," Mathematical Biosciences and Engineering, vol. 17, no. 1, pp. 179-201, 2020.

[14] T. Zhang, T. Xu, J. Wang, Y. Song, and Z. Jiang, "Geometrical analysis of a pest management model in food-limited environments with nonlinear impulsive state feedback control,"
Journal of Applied Analysis and Computation, vol. 9, no. 6, pp. 1-17, 2019.

[15] K. Gopalsamy and P. Weng, "Global attractivity in a competition system with feedback controls," Computers and Mathematics with Applications, vol. 45, no. 4-5, pp. 665-676, 2003.

[16] Z. Li, M. Han, and F. Chen, "Influence of on an autonomous Lotka-Volterra competitive system with infinite delays feedback controls," Nonlinear Analysis: Real World Applications, vol. 14, no. 1, pp. 402-413, 2013.

[17] C. Shi, X. Chen, and Y. Wang, "Feedback control effect on the lotka-volterra prey-predator system with discrete delays," Advances in Difference Equations, vol. 2017, no. 1, p. 373, 2017.

[18] F. Bian, W. Zhao, Y. Song, and R. Yue, "Dynamical analysis of a class of prey-predator model with Beddington-DeAngelis functional response, stochastic perturbation, and impulsive toxicant input," Complexity, vol. 2071, Article ID 3742197, p. 18, 2017.

[19] M. Chi and W. Zhao, "Dynamical analysis of multi-nutrient and single microorganism chemostat model in a polluted environment," Advances in Difference Equations, vol. 2018, no. 1, Article ID 120, 2018.

[20] A. Miao, T. Zhang, J. Zhang, and C. Wang, "Dynamics of a stochastic sir model with both horizontal and vertical transmission," Journal of Applied Analysis and Computation, vol. 8, no. 4, pp. 1108-1121, 2018.

[21] T. Feng, Z. Qiu, X. Meng, and L. Rong, "Analysis of a stochastic hiv-1 infection model with degenerate diffusion," Applied Mathematics and Computation, vol. 348, pp. 437-455, 2018.

[22] X. Li and X. Mao, "Population dynamical behavior of nonautonomous lotka-volterra competitive system with random perturbation," Discrete and Continuous Dynamical SystemsSeries A, vol. 24, no. 2, pp. 523-593, 2009.

[23] Y. Li and X. Meng, "Dynamics of an impulsive stochastic nonautonomous chemostat model with two different growth rates in a polluted environment," Discrete Dynamics in Nature and Society, vol. 2019, Article ID 5498569, p. 15, 2019.

[24] W. Zhang, X. Meng, and Y. Dong, "Periodic solution and ergodic stationary distribution of stochastic siri epidemic systems with nonlinear perturbations," Journal of Systems Science and Complexity, vol. 32, no. 4, pp. 1104-1124, 2019.

[25] G. Liu, Z. Chang, and X. Meng, "Asymptotic analysis of impulsive dispersal predator-prey systems with markov switching on finite-state space," Journal of Function Spaces, vol. 2019, Article ID 8057153, p. 18, 2019.

[26] H. Qi, X. Meng, and T. Feng, "Dynamics analysis of a stochastic non-autonomous one-predator-two-prey system with Beddington-DeAngelis functional response and impulsive perturbations," Advances in Difference Equations, vol. 2019, no. 1, Article ID 235, 2019.

[27] M. Chi and W. Zhao, "Dynamical analysis of two-microorganism and single nutrient stochastic chemostat model with monodhaldane response function," Complexity, vol. 2019, Article ID 8719067, p. 13, 2019.

[28] F. F. Zhu, X. Z. Meng, and T. H. Zhang, "Optimal harvesting of a competitive n-species stochastic model with delayed diffusions," Mathematical Biosciences and Engineering, vol. 16, no. 3, pp. 1554-1574, 2019.

[29] X. Yu, S. Yuan, and T. Zhang, "Persistence and ergodicity of a stochastic single species model with allee effect under regime switching," Communications in Nonlinear Science and $\mathrm{Nu}$ merical Simulation, vol. 59, pp. 359-374, 2018. 
[30] W. Zhao, J. Li, T. Zhang, X. Meng, and T. Zhang, "Persistence and ergodicity of plant disease model with markov conversion and impulsive toxicant input," Communications in Nonlinear Science and Numerical Simulation, vol. 48, pp. 70-84, 2017.

[31] W. Zhao, J. Liu, M. Chi, and F. Bian, "Dynamics analysis of stochastic epidemic models with standard incidence," $A d$ vances in Difference Equations, vol. 2019, no. 1, Article ID 22, 2019.

[32] T. Feng, Z. Qiu, and X. Meng, "Dynamics of a stochastic hepatitis c virus system with host immunity," Discrete \& Continuous Dynamical Systems-B, vol. 24, no. 12, pp. 63676385, 2019.

[33] T. Feng, Z. Qiu, and X. Meng, “Analysis of a stochastic recovery-relapse epidemic model with periodic parameters and media coverage," Journal of Applied Analysis and Computation, vol. 9, no. 3, pp. 1007-1021, 2019.

[34] N. Gao, Y. Song, X. Wang, and J. Liu, "Dynamics of a stochastic sis epidemic model with nonlinear incidence rates," Advances in Difference Equations, vol. 2019, no. 1, Article ID 41, 2019.

[35] Y. Song, A. Miao, T. Zhang, X. Wang, and J. Liu, "Extinction and persistence of a stochastic sirs epidemic model with saturated incidence rate and transfer from infectious to susceptible," Advances in Difference Equations, vol. 2018, no. 1, Article ID 293, 2018.

[36] X. Mao, Stochastic Differential Equations and Applications, Horwood Publishing, Chichester, UK, 2007.

[37] M. Liu, H. Qiu, and K. Wang, "A remark on a stochastic predator-prey system with time delays," Applied Mathematics Letters, vol. 26, no. 3, pp. 318-323, 2013. 


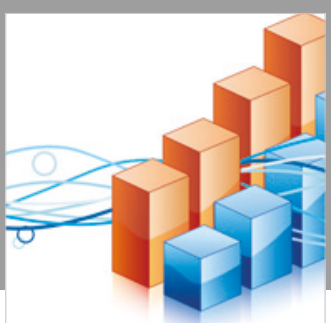

Advances in

Operations Research

\section{-n-m}
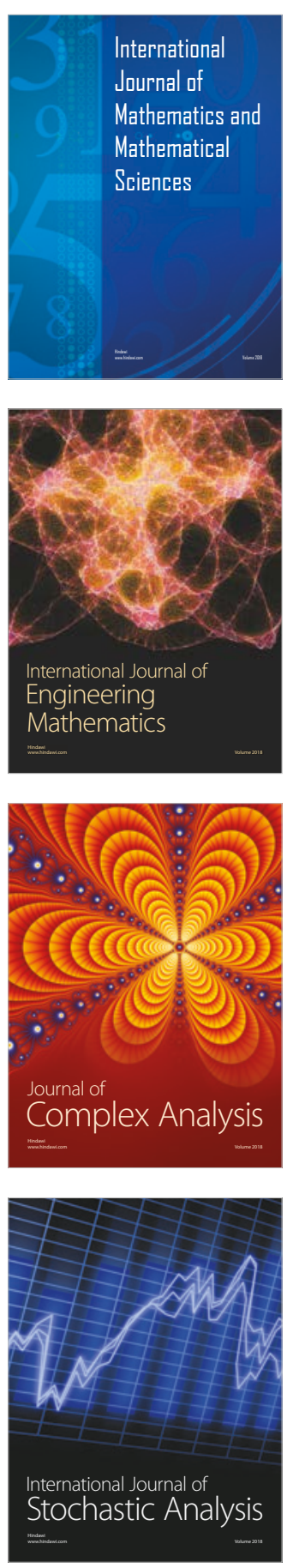
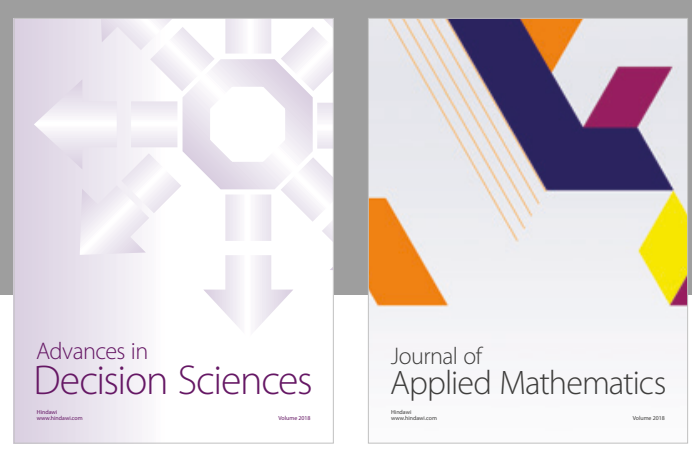

Journal of

Applied Mathematics
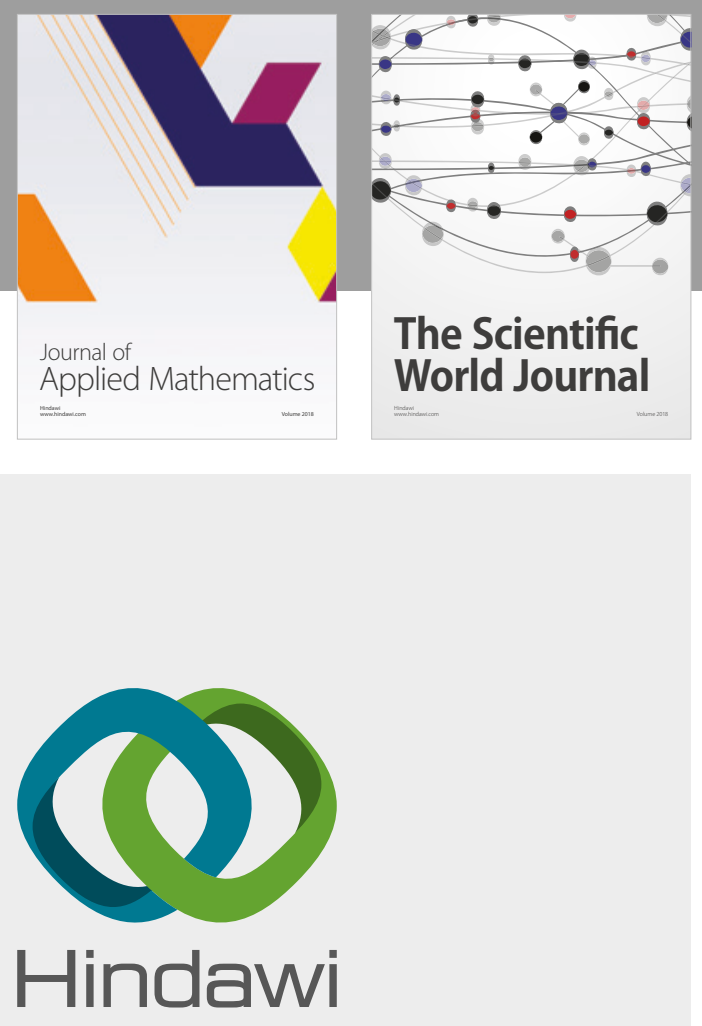

Submit your manuscripts at

www.hindawi.com

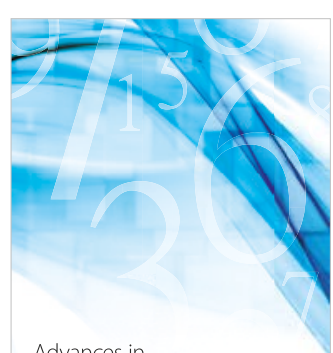

Advances in
Numerical Analysis
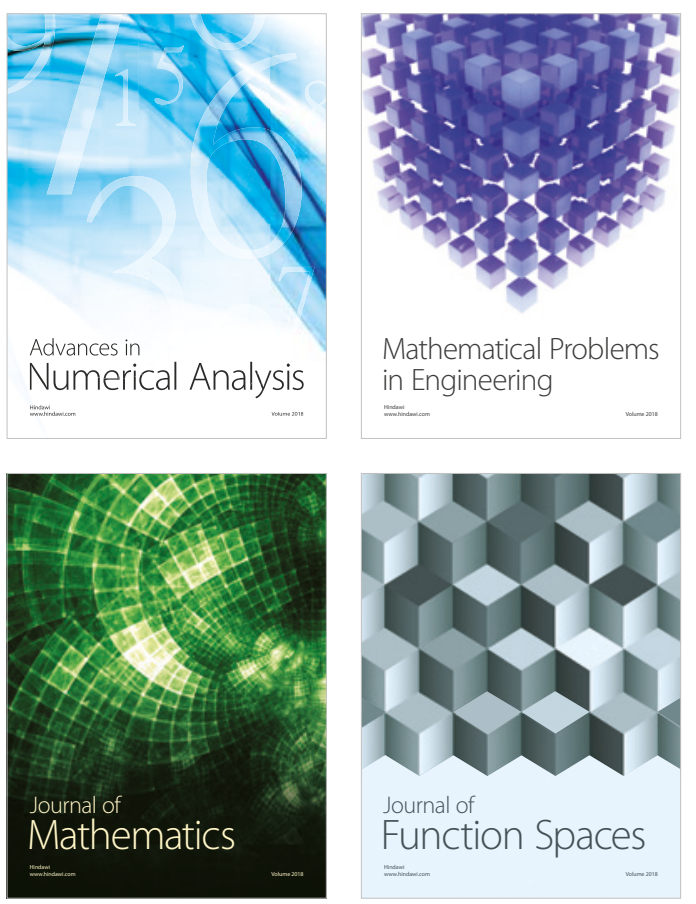

Mathematical Problems in Engineering

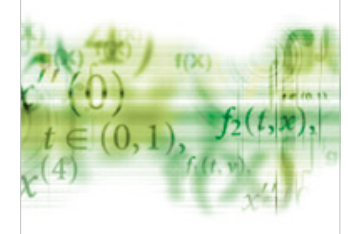

International Journal of

Differential Equations

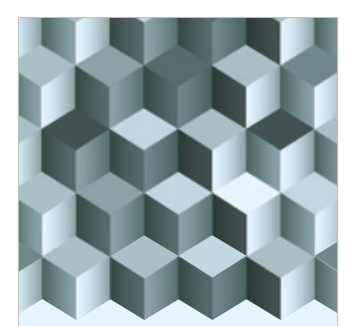

Journal of

Function Spaces
The Scientific

World Journal

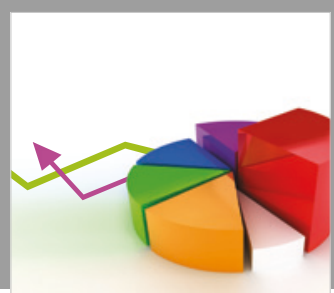

Journal of

Probability and Statistics
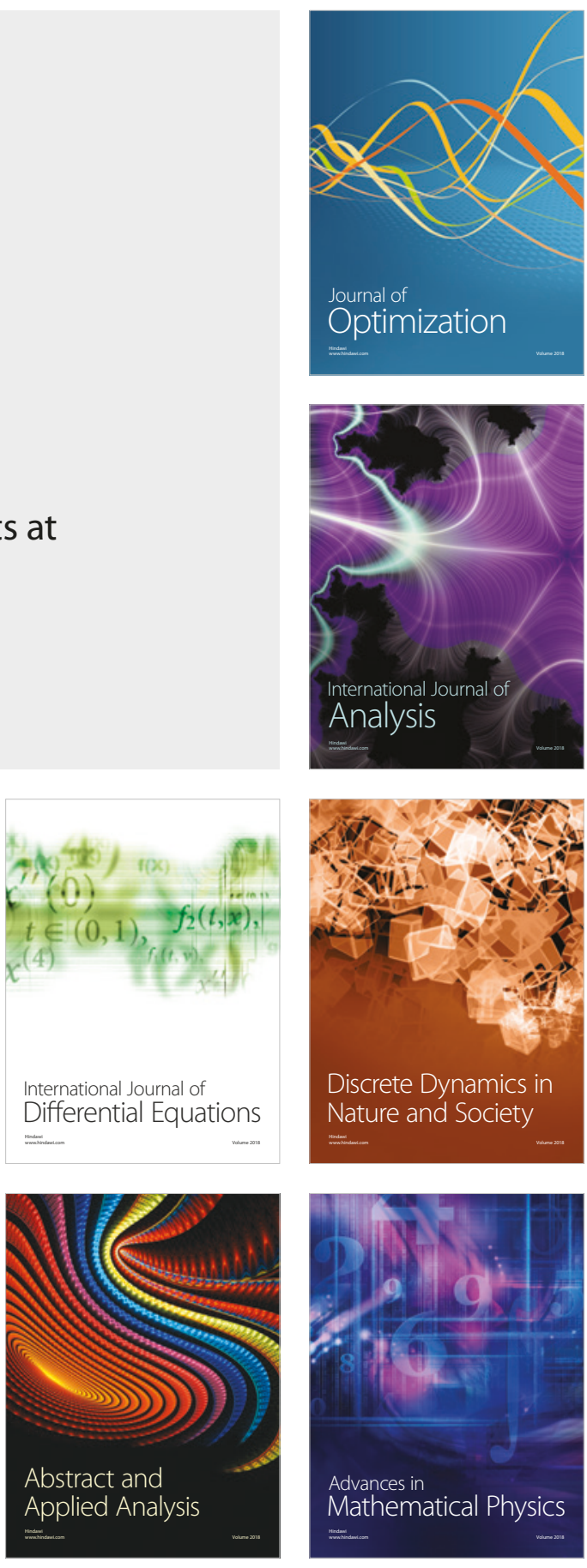